\title{
Tinetti Assessment Tool Base on Wireless Sensor Network ${ }^{*}$
}

\author{
Yundong Xuan ${ }^{1,2}$, Zhan Zhao ${ }^{1}$, Zhen Fang ${ }^{1}$, Zhihong $\mathrm{Xu}^{1,2}$ and Fangmin Sun ${ }^{1,2}$ \\ ${ }^{1}$ State Key Laboratory of Transducer Technology, Institute of Electronics, Chinese Academy of Sciences, Beijing 100190, China \\ ${ }^{2}$ University of Chinese Academy of Sciences, Beijing 100049, China \\ xuanyundong@gmail.com, \{zhaozhan \& zfang $\}$ mail.ie.ac.cn
}

\begin{abstract}
Falls are a common experience for the elderly. Falls in the elderly can be reduced by early enrollment in fall prevention programs. Identifying people at high fall risk before they have actually fallen would allow earlier enrollment in fall prevention programs and possibly prevent or delay the onset of repetitive falls. To identify those at high fall risk, measures are needed that can easily be obtained without actually inducing falls. Tinetti assessment tools used for fall-risk screening in elderly persons is based on visual observation and/or timing of physical performance. In this study we design a risk of falling assessment system based on smart shoes and we give a method to classify the items in the Tinetti Gait assessment tool table.
\end{abstract}

Index Terms - Tinetti table, Inertial sensor, Gait acceleration, Wireless sensor network.

\section{Introduction}

Falls are a common experience for the elderly. Approximately one third of people over age 65 fell each year. Although only 15 percents of these falls resulted in injuries required medical attention. Falls that result in injury increase a number of risks for the older person. Many individuals who fall develop a fear of falling. That may cause them to limit activity, leading to reduced mobility or physical fitness and increasing their risk for additional falls. The serious falls can lead to permanent impairment in functioning resulting in a major loss of independence. Falls are associated with 40 percent of nursing home admissions. Serious falls can also precipitate a cascade of events leading to death. And the rates of fall-related deaths among the elderly rose significantly over the past decade. The consequences of falls are costly in both human and fiscal terms [1].

Falls in the elderly can be reduced by early enrollment in fall prevention programs. The problem of falls among the elderly has been recognized and studied for many years, including myriad analyses regarding assessment and prevention of falls in this population. Identifying people at high fall risk before they have actually fallen would allow earlier enrollment in fall prevention programs and possibly prevent or delay the onset of repetitive falls. To identify those at high fall risk, measures are needed that can easily be obtained without actually inducing falls. Several studies suggested that increased gait variability may prospectively predict future falls [2].
Most assessment tools used for fall-risk screening in elderly persons are based on visual observation and/or timing of physical performance. Although their predictive value is undeniable, cut-off-values for clinical use merely correspond with clearly visible instability or reduced ambulation. To detect more subtle gait disturbances, instrumented assessment tool are developed, for example, gait analysis using force plates, gyroscopes, 3D accelerometers attached on the lower trunk, or combinations of the above. Several features obtained by $3 \mathrm{D}$ accelerometer signal the gait pattern such as walking speed, step and stride time, step-time asymmetry, and acceleration periods among steps or strides [2].

Table.1 Tinetti gait assessment table

\begin{tabular}{|c|c|c|}
\hline \multirow{2}{*}{$\begin{array}{l}\text { Evaluated } \\
\text { Function }\end{array}$} & \multicolumn{2}{|l|}{ Name: $\quad$ Date: } \\
\hline & Description of Behavior & score \\
\hline $\begin{array}{l}\text { Indication of } \\
\text { Gait }\end{array}$ & $\begin{array}{l}\text { Any hesitancy or multiple attempts } \\
\text { No hesitancy }\end{array}$ & $\begin{array}{l}0 \\
1\end{array}$ \\
\hline $\begin{array}{l}\text { Step length and } \\
\text { hight }\end{array}$ & $\begin{array}{l}\text { Step to } \\
\text { Step through right } \\
\text { Step through left }\end{array}$ & $\begin{array}{l}0 \\
1 \\
2\end{array}$ \\
\hline $\begin{array}{l}\text { Foot } \\
\text { Clearance }\end{array}$ & $\begin{array}{l}\text { Foot drop } \\
\text { Left foot clears the floor } \\
\text { Right foot clears the floor }\end{array}$ & $\begin{array}{l}0 \\
1 \\
2\end{array}$ \\
\hline Foot Symmetry & $\begin{array}{l}\text { Right and left step length are not equal } \\
\text { Right and left step length appear equal }\end{array}$ & $\begin{array}{l}0 \\
1\end{array}$ \\
\hline Step Continuity & $\begin{array}{l}\text { Stopping of discontinuity between steps } \\
\text { Steps appear continuous }\end{array}$ & $\begin{array}{l}0 \\
1\end{array}$ \\
\hline Path & $\begin{array}{l}\text { Marked deviation } \\
\text { Moderate deviation or uses a walking aid } \\
\text { Straight without a walking aid }\end{array}$ & $\begin{array}{l}0 \\
1 \\
2\end{array}$ \\
\hline Trunk & $\begin{array}{l}\text { Marked sway or uses a walking aid } \\
\text { No sway, flexes knees/back/uses arms to } \\
\text { balance } \\
\text { No sway, no flexion of knees or back use } \\
\text { of arms,or walking aid }\end{array}$ & $\begin{array}{l}0 \\
1 \\
2 \\
\end{array}$ \\
\hline Walking Time & $\begin{array}{l}\text { Heels apart } \\
\text { Heels almost touching while walking }\end{array}$ & $\begin{array}{l}0 \\
1\end{array}$ \\
\hline & Gait Score & \\
\hline
\end{tabular}

\footnotetext{
* This work is partially supported by NNSF of China \#60971071 and 863 HTR\&DP of China \#2009AA045300.
} 
The Tinetti assessment tools used for fall-risk screening in elderly persons is based on visual observation and/or timing of physical performance. The Tinetti gait assessment is shown in Fig.1.The items of gait assessment tool are based on 3D acceleration data when people walk. So based on acceleration data we can classify if the the elderly have the risk of falling or not and how serious the problem is.

In this study we design a risk of falling assessment system and base on the Tinetti Gait assessment we give a method to classify the items in the table.

\section{System architecture}

The Tinetti assessment tool is an easily administered taskoriented test that measures an older adult's gait and balance abilities.Fig.1 describes the architecture to implement the Tinetti tool. The architecture consists of two parts. One is the wireless sensor network which collects the inertial sensor data, and the other is The Tinetti assessment workstation which is a PC receiving data from sensor network in wireless. The wireless sensor network is made up of three nodes, one is adsorbed on the waist, and other two nodes are embedded in shoes. When the patient walks on evaluating path, the signal collected by the wireless network is transferred to the MCU in the master node in wireless. Then MCU transmits data packets to a computer running our algorithms, telling the algorithms which nodes have detected the signal. According to the coordinates of the receiving nodes, multiple data are calculated. According to the shape and the character of the signal, we can apply back the Tinetti table to identify the items of the Tinetti table through our algorithms.

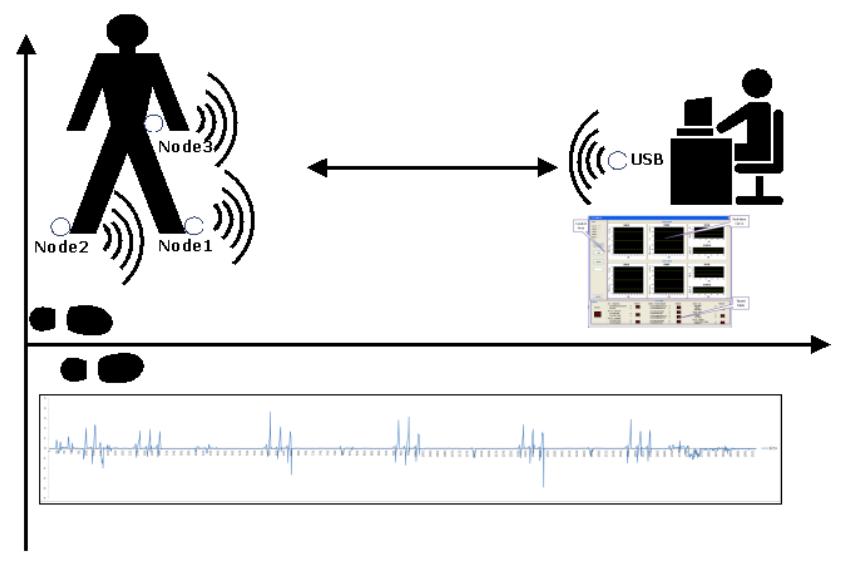

Fig.1.the system architecture of the Tinetti table

\section{Hardware of assessment system}

This section will be dedicated to the accelerometer network and the way the accelerometers are set on the body for gait analysis. The network based on Zigbee is composed of 3 slave sensor nodes and one data receiver shown in Fig.2. Each slave node is based on a Freescale MMA8451Q 3-axis accelerometer [3] and a microcontroller CC2530 with RF function from TI. The sensors are connected to the data receiver in wireless.
The accelerometers have selectable sensitivity and range between $\pm 4 \mathrm{~g}$ that the range that best fits the kind of data to be analyzed can be selected. The data receiver communicates with PC via USB, and the data is uploaded to a PC via USB port for processing and analysis. The sensors via SPI protocol and collects the 3D accelerations on each sensor at $50 \mathrm{~Hz}$. Every one node is embedded in the shoe whose blueprint is showed in Fig3. The prototype of the wearable motion detector shoes are showed in Fig4.

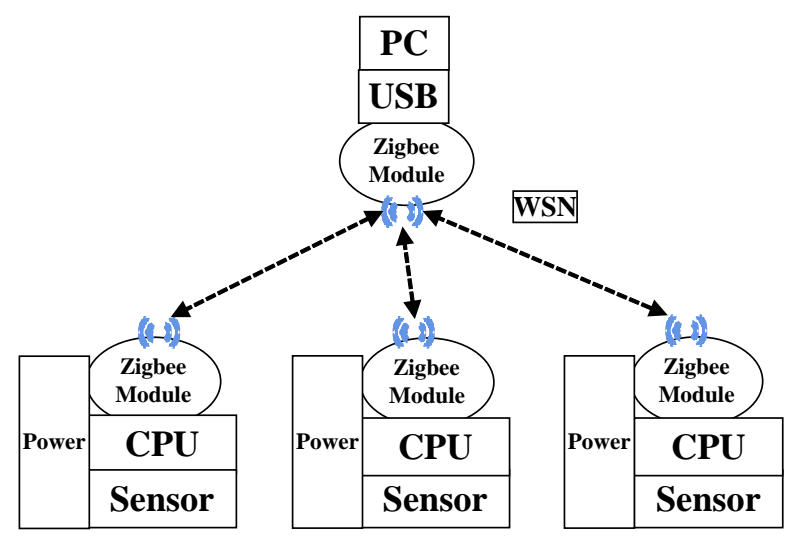

Fig.2.the hardware architecture of the assessment system

Then the data receiver sequentially collects data from each sensor. The sensors are $36 \times 22 \times 8 \mathrm{~mm}$ big and the data receiver $62 \times 22 \times 7 \mathrm{~mm}$. they are showed in Fig.5 (a) and Fig.5 (b).

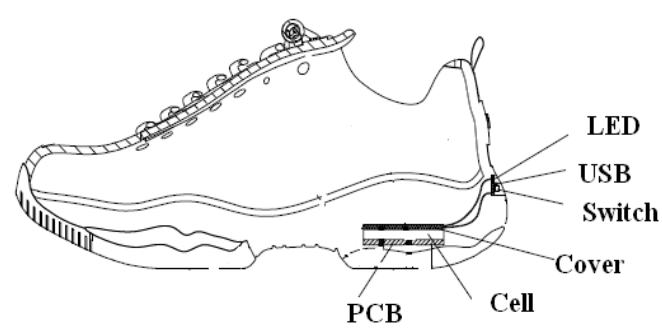

Fig.3.The system architecture of Tinetti table

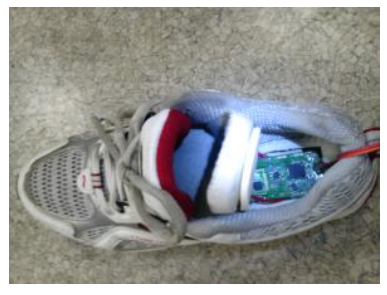

(a)

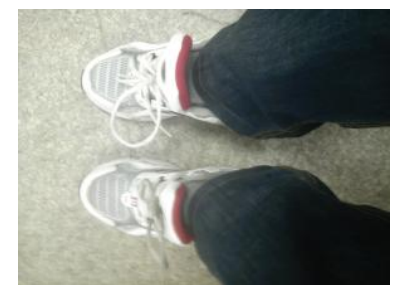

(b)
Fig.4.The prototype of the wearable sensor shoes 


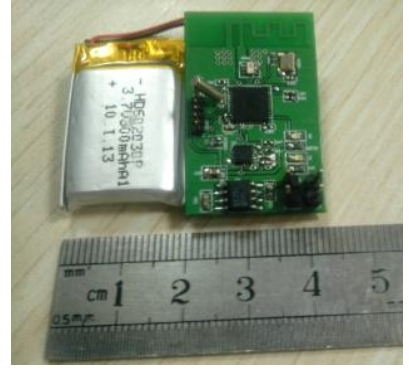

(a)

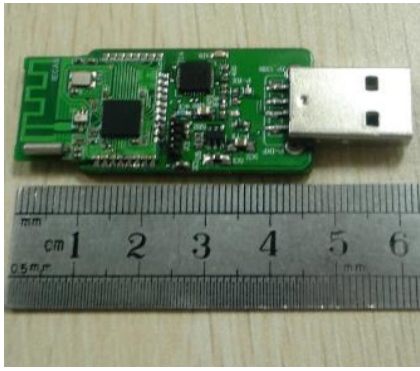

(b)
Fig.5. The devices: (a) The sensor node device; (b) The data receiver device;

\section{Software of assessment system}

Before 3D data were collected, it was impossible to use raw data to classify fallers and non-fallers. So several classification algorithms will be implemented with the aim classified fallers and non-fallers from the raw signal.

In slave sensor nodes the 3D accelerometer data is processed by filters in order to decrease the noise. The 3D accelerometer data is also encrypt and transferred to the master in wireless. The master node receives the data and uploads to PC. The data which is transferred to a PC is processed and analyzed in it.

Based on Fig. 4 depicted, The $\mathrm{X}$-axis of accelerometers is sensitive axis. So we design the algorithm shown in Fig.6 which shows a graphical representation of the basic steps of the algorithm. The signal passes through low pass filter, derivation, squaring function, Moving-Window integration, and data thresholds are set and The extremum value are detected[4,5,6,7].

In the first step the algorithm passes the signal through a low pass filter in order to reduce the influence of the wobble noise and the power line interference. After filtering, the signal is differentiated to provide the signal slope information. We use a five-point derivative.

After differentiation, the signal is squared point by point making all data point. This makes all data positive and does nonlinear amplification of the output of the derivative emphasizing the higher frequencies. After squaring, the algorithm performs moving window integration in order to obtain waveform feature information.

In the last step two thresholds are adjusted. The higher of the two thresholds is used to find out the peak value of the signal. The lower threshold is used when no peak value has been detected by the higher threshold in a certain time interval.

In this case the algorithm has to search back in time for a lost peak value. When a new peak value is identified then this peak value is classified as a signal peak value if it exceeds the high threshold or as a noise peak value otherwise.

The algorithm developed for this purpose is easy to implement, can give the peak value array real-time which can be used to estimate cadence, step regularity, stride regularity and step symmetry from the inertial signal in real time. But the fluctuation in the signal, yielding the positive and negative slopes as the useful feature, can result in false peak searching interval.

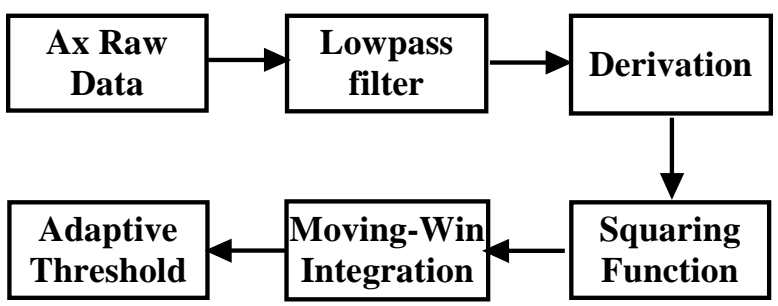

Fig.6.the system architecture of the Tinetti table

From Tinetti gait assessment tool we know there are many features extracted from the $50 \mathrm{~m}$ walking test of each subject: Indication of gait, Step length and height, Step symmetry, Step continuity, Path, Trunk, Walking time.

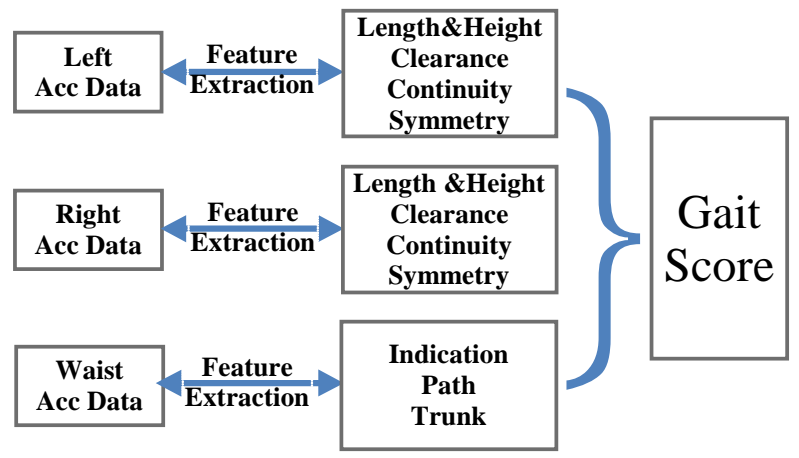

Fig.7.The software structure of the system

Based on the step above, Step length, height and Step continuity can be computed from the peak value array of the 3D accelerometer signal [8]. So the Step Length and Height and Continuity of the left foot can be computed from the accelerometer embedded in the left shoe.

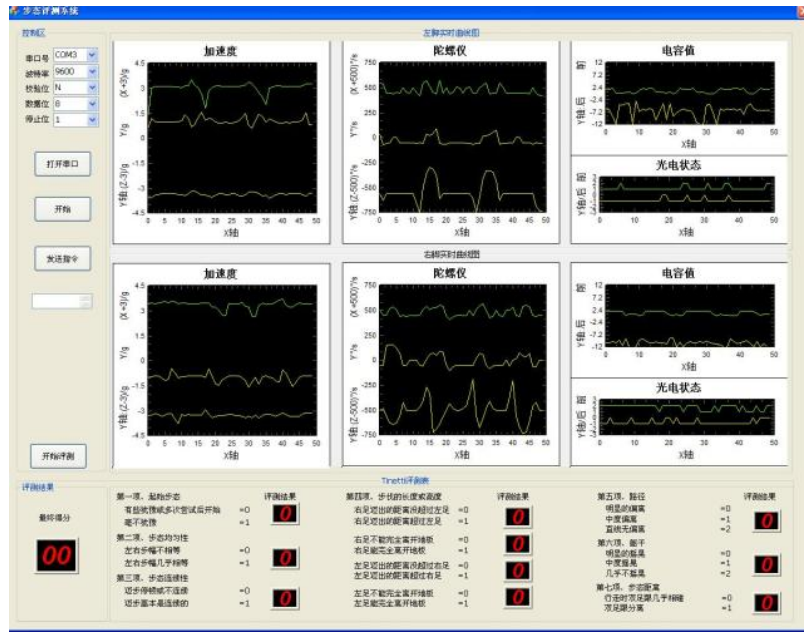

Fig.8. The Tinetti station 
And the Step Length and Height and Continuity of the right foot can be computed from the accelerometer embedded in the right shoe. The features of Indication of gait, Path and Trunk can be computed by integral data from inertial sensor adsorbed on the waist. And the Step Symmetry and Walking time can also be calculated from other features of gait peak value [9]. The software structure of the system is showed in Fig.7. The result of Tinetti assessment and the real time data are displayed on the PC as showed at Figure 8.

\section{The Conclusions and Future Work}

In this paper we design a risk of falling assessment system and base on the Tinetti Gait assessment we give a method to classify the items in the table. It is pointed out that The Tinetti Assessment Tool base on gait acceleration is viable. Many features can be computed via wireless accelerometer sensor network. Sensor-based measurements using a small wearable device may contribute significant information to conventional methods and are feasible in an unsupervised setting. More prospective research is needed to compare the predictive performance of our new sensor-based method with conventional and established methods. All these issues are planned to be addressed in the future papers.

Additionally, capacitive and photoelectric sensors will be embedded in shoes for step detection in the nearly future, which is commonly used for gait analysis

\section{Acknowledgment}

We wish to acknowledge the support of my friends at State Key Laboratory of Transducer Technology, Institute of Electronics, Chinese Academy of Sciences. This support is gratefully acknowledged.

\section{References}

[1] Marschollek et al. Sensors vs. experts - A performance comparison of sensor-based fall risk assessment vs. conventional assessment in a sample of geriatric patients. BMC Medical Informatics \& Decision Making, 2011, 11:48-55.

[2] Ivan Bautmans et al. Reliability and clinical correlates of 3Daccelerometry based gait analysis outcomes according to age and fallrisk. Gait \& Posture, 2011, 33:366-372.

[3] Freescale Devices. MMA8451Q Datasheet. http://cache.freescale.com/files/sensors/doc/data_sheet/MMA8451Q.pdf? fpsp=1, Last visit:03.02.2012.

[4] Ying, Hong; Silex, Carmen; Schnitzer, Andreas; Leonhardt, Steffen; Schiek, Michael. Automatic Step Detection in the Accelerometer Signal.BSN,2007.

[5] Pan J, Tompkins W. A real-time QRS detection algorithm[J], IEEE Transactions on Biomedical Engineering, 1985, 32: 230-236.

[6] P S Hamilton, W J Tompkins. Quantitative investigation of QRS detection rules using the MIT/BIH arrhythmia database[J]. IEEE Transactions on Biomedical Engineering, 1986, 33(12): 1157-1165.

[7] Jonghee Han, Hyo Sun Jeon, Beom Suk Jeon, Kwang Suk Park. Gait detection from three dimensional acceleration signals of ankles for the patients with Parkinson's disease.BSN,2006.

[8] Moe-Nilssen R et al. Estimation of Gait Cycle Characteristics of Trunk Accelerometry. Journal of Biomechanics, 2004, 37:121-126.

[9] Benoit Caby et al. Feature extraction and selection for objective gait analysis and fall risk assessment by accelerometry. BioMedical Engineering Online, 2011,10:1-19. 